\title{
Inguinal or inguino-iliac/obturator lymph node dissection after positive inguinal sentinel lymph node in patients with cutaneous melanoma
}

\author{
Nebojsa Glumac ${ }^{1}$, Marko Hocevar ${ }^{1}$, Vesna Zadnik ${ }^{2}$, Marko Snoj $^{1}$ \\ 1 Department of Surgical Oncology, Institute of Oncology, Ljubljana, Slovenia \\ ${ }^{2}$ Cancer Registry, Institute of Oncology, Ljubljana, Slovenia
}

Radiol Oncol 2012; 46(3): 258-264.

Received 22 August 2011

Accepted 11 September 2011

Correspondence to: Nebojša Glumac, MD, PhD, Department of Surgical Oncology, Institute of Oncology, Zaloška 2, 1000 Ljubljana, Slovenia. Phone: +386 41437 890; Fax: +386 15879 407; E-mail: nglumac@onko-i.si

Disclosure: No potential conflicts of interest were disclosed.

Background. The aim of the study was to determine whether the presence of inguinal sentinel lymph node (SLN) metastases smaller than $2 \mathrm{~mm}$ (micrometastases) subdivided according to the number of micrometastases predicts additional, non-sentinel inguinal, iliac or obturator lymph node involvement in completion lymph node dissection (CLND). Patients and methods. Positive inguinal SLN was detected in 58 patients ( 32 female, 26 male, median age 55 years) from 743 consecutive and prospectively enrolled patients with primary cutaneous melanoma stage I and II who were treated with SLN biopsy between 2001 and 2007.

Results. Micrometastases in inguinal SLN were detected in 32 patients, 14 were single, 2 were double, and 16 were multiple. Twenty-six patients had macrometastases.

Conclusions. No patient with any micrometastases or a single SLN macrometastasis in the inguinal region had any iliac/obturator non-sentinel metastases after CLND in our series. Furthermore, no patient with single SLN micrometastasis in the inguinal region had any non-sentinel metastases at all after CLND in our series. In these cases respective CLND might be omitted.

Key words: melanoma; inguinal; sentinel lymph node biopsy; micrometastases; lymphadenectomy

\section{Introduction}

Since its introduction by Morton in 1992, sentinel lymph node biopsy (SLNB) is now becoming a standard of care for staging of patients with clinical stage I and II cutaneous melanoma or breast cancer. ${ }^{1-3}$ Sentinel lymph node (SLN) predicts the status of regional nodal basin and its surgical recovery nears $100 \%{ }^{4}$ The standard of treatment for positive SLN is completion lymph node dissection (CLND). ${ }^{5}$ Specifically, in the inguinal region the extent of CLND is not yet clearly defined. Both inguinal and inguino-iliac/obturator lymph node dissection are being performed.
Before the introduction of SLNB the standard of treatment for palpable metastatic inguinal lymph nodes in patients with cutaneous melanoma was inguino-iliac/obturator lymph node dissection. This was due to the fact that up to $40 \%$ of patients with palpable metastatic inguinal lymph nodes had metastases in the iliac or obturator nodes. ${ }^{6}$

Nowadays, the situation has changed. The metastatic deposits found in the SLN are much smaller than previously detected palpable metastases and thus the chance of iliac or obturator node metastasis is considerably lower. Frequently, the CLND after positive inguinal SLN is completely negative thus adding the patient only additional complica- 
tions and morbidity, and no benefit. . $^{4,7}$ Namely, only $10-30 \%$ of patients with SLN metastases in any region have additional metastases in non-sentinel lymph nodes. ${ }^{8}$ There are even fewer patients with iliac or obturator metastases after positive inguinal SLN. ${ }^{9}$ Therefore, inguinal dissection might be radical enough procedure after positive inguinal SLN. However, at present time, it is difficult to predict in which subset of inguinal SLN positive patients the iliac/obturator lymph node dissection would not present with any further positive lymph nodes. Through the literature review we did not find any author who dealt specifically with the pattern of non-sentinel metastasis after a positive inguinal SLN.

The aim of our study was to determine the predictive value of inguinal SLN micrometastases subdivided according to the number of micrometastases in predicting non-sentinel inguinal, iliac or obturator lymph node involvement in CLND. In addition, we reviewed files of melanoma patients who were surgically treated for palpable inguinal metastases in the same time period.

\section{Patients and methods}

\section{Patients}

The files of all 743 consecutive and prospectively enrolled patients with primary cutaneous melanoma stage I and II who were treated with SLNB at the Institute of Oncology, Ljubljana, Slovenia between January 2001 and December 2007 were reviewed retrospectively in our study. Positive inguinal SLN was detected in 58 patients ( 32 female, 26 male, median age 55 years). In addition, we reviewed files of 94 stage III melanoma patients who were surgically treated for palpable inguinal metastases at our department in the same time period.

The investigators strictly followed recommendations of the Helsinki declaration and of the European Council Convention on Protection of Human Rights in Bio-Medicine.

\section{Methods}

Preoperatively, ultrasound of the inguinal region was performed in most patients. Criteria for SLNB were as follows: cutaneous melanoma stage I or II thicker than $1 \mathrm{~mm}$ or thinner than $1 \mathrm{~mm}$, but with signs of gross regression, ulceration or Clark level IV/V. SLNB and wide local excision were performed using the standard triple technique of preoperative lymphoscintigraphy using ${ }^{99 \mathrm{~m}} \mathrm{Tc}$ ra-
TABLE 1. Patients' clinicopathological characteristics after positive inguinal SLNB

\begin{tabular}{|c|c|}
\hline Characteristic & SLNB group \\
\hline Total, $\mathrm{n}$ & 58 \\
\hline \multicolumn{2}{|l|}{ Sex, n (\%) } \\
\hline Female & $32(55.2 \%)$ \\
\hline Male & $26(44.8 \%)$ \\
\hline \multicolumn{2}{|l|}{ Age, years } \\
\hline Median & 55 \\
\hline Range & 7.6-87.1 \\
\hline \multicolumn{2}{|l|}{ Primary site, $\mathrm{n}(\%)$} \\
\hline Trunk & 11 (19\%) \\
\hline Lower extremity & $47(81 \%)$ \\
\hline Unknown & $0(0 \%)$ \\
\hline \multicolumn{2}{|l|}{ Breslow, mm } \\
\hline Mean & 3.66 \\
\hline Median & 3.2 \\
\hline Range & $1.1-13$ \\
\hline $0-1, n(\%)$ & $0(0 \%)$ \\
\hline $1.01-2, \mathrm{n}(\%)$ & 14 (24.1\%) \\
\hline $2.01-4, n(\%)$ & 30 (51.8\%) \\
\hline$>4.01, \mathrm{n}(\%)$ & $14(24.1 \%)$ \\
\hline Unknown & $0(0 \%)$ \\
\hline \multicolumn{2}{|l|}{ Clark, n (\%) } \\
\hline III & $11(19 \%)$ \\
\hline IV & $36(62 \%)$ \\
\hline $\mathrm{V}$ & $4(6.9 \%)$ \\
\hline Unknown & $7(12.1 \%)$ \\
\hline \multicolumn{2}{|l|}{ Ulceration, n (\%) } \\
\hline Yes & $30(51.7 \%)$ \\
\hline No & 21 (36.2\%) \\
\hline Unknown & $7(12.1 \%)$ \\
\hline \multicolumn{2}{|l|}{ SLN removed, $\mathrm{n}$} \\
\hline Mean & 1.86 \\
\hline Range & $1-4$ \\
\hline SLN status, n (\%) & 14 (24.1\%) \\
\hline 1 micrometastasis & $2(3.5 \%)$ \\
\hline 2 micrometastases & $16(27.6 \%)$ \\
\hline $\begin{array}{l}\text { 2+ micrometastases } \\
\text { Macrometastases }\end{array}$ & $26(44.8 \%)$ \\
\hline \multirow{2}{*}{\multicolumn{2}{|c|}{ CLND nodes removed, $\mathrm{n}$ (mean) }} \\
\hline & \\
\hline Inguinal dissection & 10.1 \\
\hline Inguino-iliac dissection & 19.3 \\
\hline
\end{tabular}

SLNB = sentinel lymph node biopsy; $S L N=$ sentinel lymph node; $C L N D=$ completion lymph node dissection

diolabelled nano-colloid, followed by perioperative intradermal injection of Patent blue dye and intraoperative use of a hand-held sterile gamma probe. ${ }^{10}$ SLN was defined as the only hot and/or blue node, the hot and/or blue node receiving afferent lymphatic channel from the tumour and the hot and/or blue node which was the first one in sequential pattern.

The SLN was examined histopatologically by using routine $\mathrm{HE}$ and immunohistochemical stain- 
TABLE 2. Patients' clinicopathological characteristics after palpable inguinal metastases

\begin{tabular}{|c|c|}
\hline Characteristic & $\begin{array}{l}\text { Palpable } \\
\text { metastases }\end{array}$ \\
\hline Total, $n$ & 93 \\
\hline \multicolumn{2}{|l|}{ Sex, n (\%) } \\
\hline Female & $53(57 \%)$ \\
\hline Male & $40(43 \%)$ \\
\hline \multicolumn{2}{|l|}{ Age, years } \\
\hline Median & 66 \\
\hline Range & $18-75$ \\
\hline \multicolumn{2}{|l|}{ Primary site, n (\%) } \\
\hline Trunk & $9(10 \%)$ \\
\hline Lower extremity & 71 (76\%) \\
\hline Unknown & $13(14 \%)$ \\
\hline \multicolumn{2}{|l|}{ Breslow, mm } \\
\hline Mean & 4.58 \\
\hline Median & 3.45 \\
\hline Range & $0.5-25$ \\
\hline $0-1, n(\%)$ & $7(7 \%)$ \\
\hline $1.01-2, \mathrm{n}(\%)$ & 12 (13\%) \\
\hline $2.01-4, n(\%)$ & $22(24 \%)$ \\
\hline$>4.01, \mathrm{n}(\%)$ & $26(28 \%)$ \\
\hline Unknown & $26(28 \%)$ \\
\hline \multicolumn{2}{|l|}{ Clark, n (\%) } \\
\hline III & $14(15 \%)$ \\
\hline IV & $34(37 \%)$ \\
\hline $\mathrm{V}$ & $7(7 \%)$ \\
\hline Unknown & $33(36 \%)$ \\
\hline \multicolumn{2}{|l|}{ Ulceration, n (\%) } \\
\hline Yes & $26(28 \%)$ \\
\hline No & $9(10 \%)$ \\
\hline Unknown & $58(62 \%)$ \\
\hline \multicolumn{2}{|l|}{ Palpable nodes positive, $\mathrm{n}$} \\
\hline Mean & 3.45 \\
\hline Range & $2-26$ \\
\hline \multicolumn{2}{|c|}{ LND nodes removed, $\mathrm{n}$ (mean) } \\
\hline Inguinal dissection & 12.9 \\
\hline Inguino-iliac dissection & 16.7 \\
\hline
\end{tabular}

LND = lymph node dissection

ing for S-100 protein and HMB-45 antigen as previously reported..$^{10}$ Briefly, SLN were bisected, fixed in formalin and embedded in paraffin. A maximum of 8 sections of each half were made, odd sections were stained with routine $\mathrm{HE}$ and even sections were immunohistochemically stained for S-100 except for the section 6 which was immunohistochemically stained for HMB45. For the purpose of our study the definitions of micrometastases and macrometastases are metastatic deposits within SLN smaller or equal to $(\leq) 2 \mathrm{~mm}$ and larger than (>) $2 \mathrm{~mm}$, respectively. We acknowledge that this is in contrast to the current TNM classification where micrometastases and macrometastases are defined as clinically non palpable and palpable metastat- ic nodes respectively. ${ }^{11}$ A cluster of cells (10 - 30 grouped cells) or isolated tumour cells (up to 20 individual cells) were also defined as micrometastasis. ${ }^{12}$ The number of micrometastases and macrometastases was also recorded.

Every patient with metastatic inguinal LN underwent a (C)LND of the involved nodal basin regardless of the metastasis size (palpable or non-palpable, identified at the SLNB) or number. Inguinal or inguino-iliac/obturator lymph node dissection was performed depending on surgeon's personal decision, guided mostly by the age and performance status of the patient. The metastatic involvement of non-sentinel inguinal, iliac or obturator lymph nodes was recorded. The nodes in the CLND specimens were evaluated by the routine $\mathrm{HE}$ technique. Adjuvant radiotherapy was given to the patients with more than $3 \mathrm{~cm}$ metastatic nodes, with more than 3 metastatic nodes or with metastatic extra capsular extension.

\section{Statistical analysis}

Numerical variables are presented by its mean, median, followed by minimum and maximum values. Attributive data are described as the absolute numbers with corresponding relative frequencies. The overall survival rates were analysed using Kaplan-Meier method..$^{13}$ Log-rank test was used to test the equality of the overall survival curves from the time of diagnosis until death of any cause. Chisquared test was used to determine the statistical significance of the association between inguinal SLN histology and CLND status. All tests were two-sided. A $p$ value of less than 0.05 was considered statistically significant. The statistical analysis was performed using the SPSS/PC software package (version 13.0. for Windows).

\section{Results}

\section{Inguinal metastases detected after SLNB}

Between January 2001 and December 2007, all lymphatic basins SLNB were performed in 743 patients. Overall, positive inguinal SLN was detected in 58 cases. Median follow-up of these SLN positive patients was 1.87 years (range 0.76-7.17). Clinicopathological characteristics of the patients with positive inguinal SLN are given in Table 1 and additional data for 93 patients with palpable inguinal metastases are given in Table 2.

Twenty-six (44.8\%) patients had macrometastases in the inguinal SLN. Micrometastases (includ- 
TABLE 3. Association between CLND negative and positive patients divided according to micrometastases and macrometastases

\begin{tabular}{lccc}
\hline & CLND negative & CLND positive & Total \\
\hline micrometastases & 31 & 1 & 32 \\
macrometastases & 16 & 10 & 26 \\
Total & 47 & 11 & 58 \\
\hline
\end{tabular}

Chi-square, $p=0.01 ; C L N D=$ completion lymph node dissection

TABLE 4. Studies reporting on SLN characteristics predictive for the absence of additional metastases in non-sentinel lymph nodes

\begin{tabular}{|c|c|c|}
\hline Author & $\mathbf{N}$ & SLN characteristic(s) \\
\hline Carlson et al. $2003^{14}$ & 104 & Not found \\
\hline Cochran et al. 200417 & 90 & Tumour area $<1 \%$ \\
\hline Dewar et al. $2004^{18}$ & 146 & Subcapsular location \\
\hline Vuylsteke et al. $2005^{19}$ & 71 & Breslow $<2.5 \mathrm{~mm}$, tumour load $<0.3 \mathrm{~mm}^{2}$ \\
\hline van Akkooi et al. $2006^{16}$ & 77 & Micrometastases $<0.1 \mathrm{~mm}$ \\
\hline Glumac et al. $2008^{7}$ & 74 & Single micrometastasis $<2 \mathrm{~mm}$ \\
\hline
\end{tabular}

$\mathrm{N}=$ Number of patients with positive SLN; SLN = sentinel lymph node

ing clusters of cells and isolated tumour cells) in inguinal SLN were detected in 32 (55.2\%) patients.

The patients with micrometastases were divided into three groups on the basis of the micrometastases number in the inguinal SLN: there were 14 patients with single micrometastasis, 2 patients with double micrometastases in one node, and 16 patients with more than two micrometastases in one or more lymph nodes (Table 1). Inguinal or inguino-iliac/obturator CLND was performed in all patients depending on surgeon's personal decision, guided mostly by the age and performance status of the patient.

Out of 14 patients with solitary and 2 patients with double micrometastases, all were without additional metastases in non-sentinel lymph nodes regardless of the type of CLND performed. Out of 16 patients with 3 or more micrometastases, only 1 had additional positive non-sentinel lymph nodes. Altogether, there were 11 patients with metastases in non-sentinel lymph nodes; 1 had multiple micrometastases in the inguinal SLN and 10 had macrometastases in the inguinal SLN. The difference between CLND negative and positive groups of patients, divided according to inguinal SLN tumour burden, was statistically significant with Chi-square $p$ value of 0.01 (Table 3 ).

Inguinal CLND was performed in 40/58 (69\%) patients. The median follow up in this group was 1.59 years (range 0.76-5.69). Seven patients had positive non-sentinel nodes (5 patients had 1 posi- tive non-sentinel node, 1 patient had 2 and 1 patient had 4 positive non-sentinel nodes). Out of those 7 patients, 6 were disease free upon follow up and 1 died with gross melanosis of the leg without systemic progress.

Inguino-iliac/obturator CLND was performed in $18 / 58(31 \%)$ patients. The median follow up of these patients was 2.71 years (range 0.83-7.17). Four patients had positive non-sentinel nodes (3 patients had 1 positive non-sentinel node and 1 patient had 5 positive non-sentinel nodes). Out of those 4 patients, 3 were disease free upon follow up and 1 died from systemic progress.

Adjuvant postoperative radiotherapy was given to 2 patients (3.5\%). Additional 17 patients $(29.3 \%)$ received palliative radiotherapy later in time due to disease progression.

Not surprisingly, the log rank test of KaplanMeier survival curves showed a statistically significant better survival (Figure 1, $p=0.032$ ) for patients with SLN micrometastases (91.5\% overall survival at 2 years, CI $84.1 \%$ - 98.9\%, median follow up 2.5 years) compared to patients with SLN macrometastases (64.0\% overall survival at 2 years, CI 50.3\% - $77.7 \%$, median follow up 1.6 years).

On the other hand, there was no statistical difference after log rank test of Kaplan-Meier survival curves ( $p=0.604$ ) for patients after inguinal CLND compared to patients after inguino-iliac/obturator CLND. 


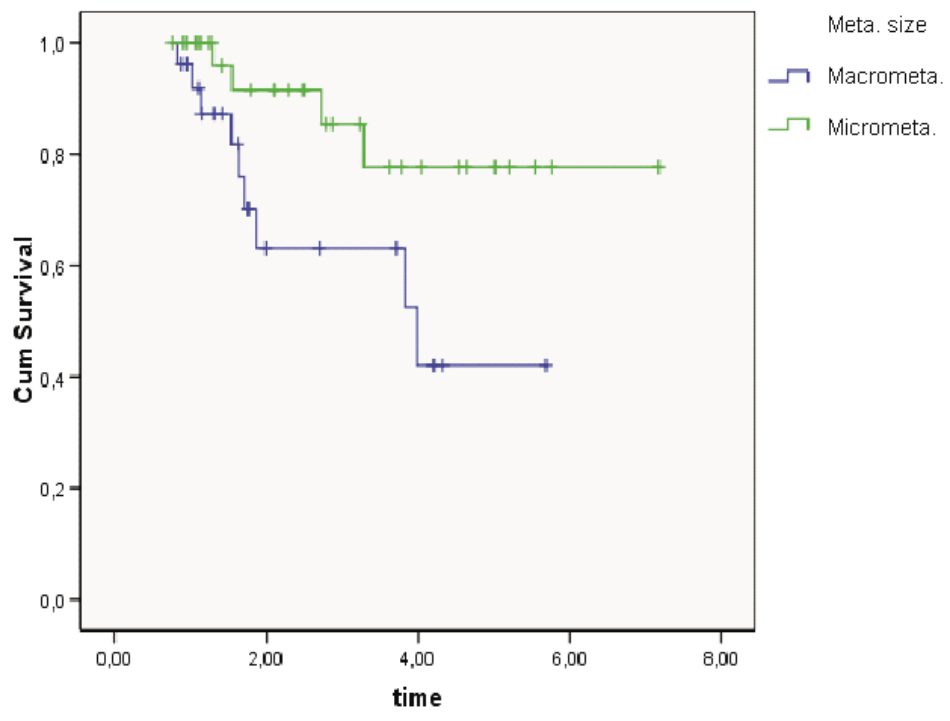

SLN = sentinel lymph node

FIGURE 1. The log rank test of Kaplan-Meier overall survival curves for patients with SLN micrometastases compared to patients with SLN macrometastases ( $p=0.032$ ).

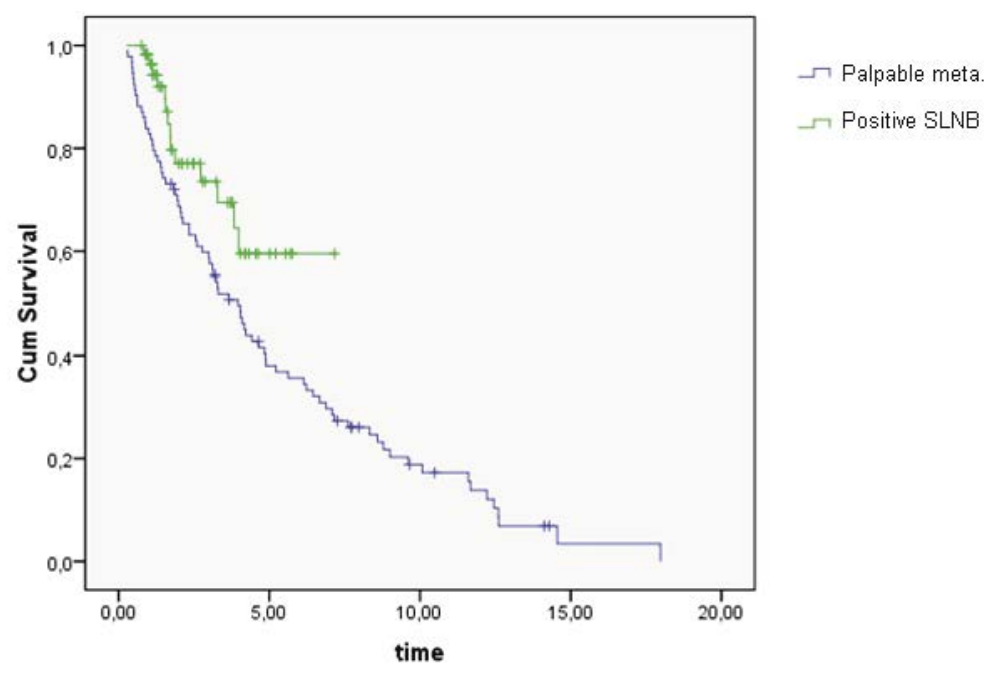

SLN = sentinel lymph node

FIGURE 2. The log rank test of Kaplan-Meier overall survival curves for patients after positive inguinal SLN compared to patients after palpable inguinal metastases $(p=0.028)$.

\section{Palpable inguinal metastases}

In addition, there were 93 stage III melanoma patients who were surgically treated for palpable inguinal metastasis in the same time period. Inguinal LND was performed in 21/93 (23\%) patients while inguino-iliac/obturator LND was performed in $72 / 93$ (77\%). There was not any statistical difference in the log rank test of Kaplan-Meier survival curves $(p=0.420)$ between patients when compar- ing the type of dissection performed after palpable inguinal metastases.

On average, there were 3.45 positive LN after palpable inguinal metastases while there were only 1.28 positive $\mathrm{LN}$ after positive inguinal SLNB (Tables 1 and 2). There were 21/93 (22.6\%) patients with positive iliac/obturator LN after palpable inguinal metastases while there were only $3 / 58$ $(5.2 \%)$ patients with positive iliac/obturator LN after positive inguinal SLNB.

The log rank test of Kaplan-Meier survival curves showed a statistically significant better overall survival (Figure 2, $p=0.028$ ) for patients with positive inguinal SLNB $(77.1 \%$ survival at 2 years, CI $64.4 \%-89.8 \%$, median follow up 1.9 years) than for patients with palpable inguinal metastases (70.5\% survival at 2 years, CI $60.3 \%-807 \%$, median follow up 3.3 years).

\section{Discussion}

As we have shown in our previous study, metastases in non-sentinel lymph nodes in patients with micrometastases in SLN are a rare event regardless of the lymphatic region. In fact, no patient with a single SLN micrometastasis in any region had metastases in CLND. ${ }^{7}$

Our study focused on the rates of inguinal, iliac and obturator non-sentinel metastatic involvement in relation to micrometastases, macrometastases and number of micrometastases in the inguinal SLN.

Due to CLND associated morbidity, such as scarring, limb oedema, seroma formation, paresthesias and deterioration of pre-existing medical co-morbidities, identification of patients without non-sentinel lymph node involvement would be of great clinical importance. Specifically in the inguinal region, the pattern of non-sentinel lymph node involvement of inguinal, iliac and obturator nodes is of great interest, due to possibility of omitting the iliac/obturator part of the CLND.

We observed that more than half $(55.2 \%)$ of the positive SLN were micrometastatic, which presents a rather high percentage, yet similar to the results reported by other authors. ${ }^{14}$ This fact might be explained by the selection of patients because the majority of our patients underwent preoperative ultrasound of the inguinal region, thus detecting at least some larger metastases that are otherwise too small to be palpated.

In 14 cases, SLN micrometastases were single, while in 2 they were double, and in 16 multiple. We observed that no patient with any microme- 
tastases or a single SLN macrometastasis had any iliac/obturator non-sentinel metastases after CLND. Furthermore, no patient with single SLN micrometastasis had any non-sentinel metastases at all after CLND. Statistically, the difference in finding additional positive nodes after CLND between the groups with inguinal SLN micrometastases and macrometastases was statistically significant $(p=$ 0.01 ) confirming the fact that the obvious difference between the mentioned groups is not a coincidence.

Iliac/obturator CLND might be avoided after detecting a single macrometastasis or any micrometastases in the inguinal SLN and CLND of any type might be avoided after detecting a single micrometastasis in the inguinal SLN. These statements are additionally supported (although clearly not proven) by the absence of statistically significant survival difference between Kaplan-Meier survival curves $(p=0.604)$ for patients after inguinal CLND compared to patients after inguino-iliac/obturator CLND in the SLNB positive patients and even in patients with palpable inguinal melanoma metastases. Another fact supporting our proposal for omitting the iliac/obturator part of CLND after micrometastases in SLN is that out of 7 patients with additional metastases after only inguinal CLND, 6 were disease free upon last follow up and 1 died with gross melanosis of the leg without systemic progress.

On the other hand, we found iliac/obturator metastases in $21 \%$ of patients with palpable inguinal melanoma metastases and there was statistical difference in survival between patients with positive inguinal SLNB and palpable inguinal metastases (Figure 2). Hence, full inguino-iliac/obturator LND is still recommended after finding palpable inguinal metastases.

One of the first researchers to deal with the subject of non-sentinel metastases was Starz et al. in $2001 .^{15}$ His group tried to predict non-sentinel node involvement by creating so called "S classification" using two morphometric parameters: the number of tumour-involved, 1-mm slices of the SLN and the centripetal depth of metastatic cell invasion. Their otherwise quite complicated system of classification has shown that there are no further metastases when SLN metastasis is less or equal to $1 \mathrm{~mm}$ and located peripherally. The Eggermont study revealed a group of patients with sub-micrometastases $(<0.1 \mathrm{~mm})$ that had no nonsentinel metastases and are thus unlikely to benefit from CLND. ${ }^{16}$ Similarly, other authors are trying to determine which SLN characteristics prognosticate no further metastases to non-sentinel lymph nodes
(Table 4). Vuylsteke et al. found this to be primary cutaneous melanoma with Breslow thickness of 2.5 $\mathrm{mm}$ or less and the surface of metastases in SLN of $0.3 \mathrm{~mm}^{2}$ or less. ${ }^{19}$ Dewar et al. described it as only subcapsular localisation of metastases in $\mathrm{SLN}^{18}$ and Cochran et al. reported it as a relative metastases surface to SLN surface of $1 \%$ or less. ${ }^{17}$ In contrast, Carlson et al. were unable to predict no involvement to non-sentinel lymph nodes by any known parameter. ${ }^{14}$ The difference between Carlson's study and ours is that we subdivided micrometastases to single, double or multiple micrometastases that yielded the group of single micrometastasis that had no additional metastases in non-sentinel lymph nodes. Through the literature review we did not find any author who dealt specifically with the pattern of non-sentinel metastasis after a positive inguinal SLN.

At present, we feel that, after micrometastases in the inguinal SLN are detected, iliac/obturator CLND can be omitted. However, this question needs to be addressed in a properly designed prospective trial.

\section{References}

1. Morton DL, Wen DR, Wong JH, Economou JS, Cagle LA, Storm FK, et al. Technical details of intraoperative lymphatic mapping for early stage melanoma. Arch Surg 1992; 127: 392-9.

2. Momennezhad M, Zakavi SR, Kakhki VRD, Jangjoo A, Ghavamnasiri MR, Sadeghi R. Scatterogram: a method for outlining the body during lymphoscintigraphy without using external flood source. Radiol Oncol 2011; 45: 184-8.

3. Polom K, Murawa D, Michalak M, Murawa P. Sentinel node biopsy in breast cancer using infrared laser system first experience with PDE camera. Rep Pract Oncol Radiother 2011; 16: 82-6.

4. Cochran AJ, Balda BR, Starz H, Bachter D, Krag DN, Cruse CW, et al. The Augsburg Consensus. Techniques of lymphatic mapping, sentinel lymphadenectomy, and completion lymphadenectomy in cutaneous malignancies. Cancer 2000; 89: 236-41.

5. Pilko G, Zgajnar J, Music M, Hocevar M. Lower tumour burden and better overall survival in melanoma patients with regional lymph node metastases and negative preoperative ultrasound. Radiol Oncol 2012; 46(3): 258-264.; 46: $60-8$.

6. Hughes TM, A'Hern RP, Thomas JM. Prognosis and surgical management of patients with palpable inguinal lymph node metastases from melanoma. $\mathrm{Br}$ J Surg 2000; 87(7): 892-901.

7. Glumac N, Hocevar M, Zadnik V, Snoj M. Sentinel lymph node micrometastasis may predict non-sentinel involvement in cutaneous melanoma patients. J Surg Oncol 2008; 98: 46-8.

8. McMasters KM, Wong SL, Edwards MJ, Chao C, Ross MI, Noyes RD, et al. Frequency of nonsentinel lymph node metastasis in melanoma. Ann Surg Oncol 2002; 9: 137-41.

9. van der Ploeg IM, Kroon BB, Valdés Olmos RA, Nieweg OE. Evaluation of lymphatic drainage patterns to the groin and implications for the extent of groin dissection in melanoma patients. Ann Surg Oncol 2009; 16: 2994-9.

10. Hocevar M, Bracko M, Pogacnik A, Vidergar-Kralj B, Besic N, Zgajnar J. Role of imprint cytology in the intraoperative evaluation of sentinel lymph nodes for malignant melanoma. Eur J Cancer 2003; 39: 2173-8. 
11. Balch CM, Buzaid AC, Soong SJ, Atkins MB, Cascinelli N, Coit DG, et al. Final version of the American Joint Committee on Cancer staging system for cutaneous melanoma. J Clin Oncol 2001; 19: 3635-48.

12. Hermanek P, Hutter RV, Sobin LH, Wittekind C. International Union Against Cancer. Classification of isolated tumor cells and micrometastasis. Cancer 1999; 86: 2668-73.

13. Kaplan EL, Meier P. Non-parametric estimation for incomplete observations J Am Stat Assoc 1958; 53: 457-81

14. Carlson GW, Murray DR, Lyles RH, Staley CA, Hestley A, Cohen C. The amount of metastatic melanoma in a sentinel lymph node: does it have prognostic significance? Ann Surg Oncol 2003; 10: 575-81.

15. Starz H, Balda BR, Krämer KU, Büchels H, Wang H. A micromorphometrybased concept for routine classification of sentinel lymph node metastases and its clinical relevance for patients with melanoma. Cancer 2001; 91: 2110-21.

16. van Akkooi AC, de Wilt JH, Verhoef $C$, Schmitz PI, van Geel AN, Eggermont $\mathrm{AM}$, et al. Clinical relevance of melanoma micrometastases $(<0.1 \mathrm{~mm})$ in sentinel nodes: are these nodes to be considered negative? Ann Oncol 2006; 17: 1578-85.

17. Cochran AJ, Wen DR, Huang RR, Wang HJ, Elashoff R, Morton DL. Prediction of metastatic melanoma in nonsentinel nodes and clinical outcome based on the primary melanoma and the sentinel node. Mod Pathol 2004; 17 747-55.

18. Dewar DJ, Newell B, Green MA, Topping AP, Powell BW, Cook MG. The microanatomic location of metastatic melanoma in sentinel lymph nodes predicts nonsentinel lymph node involvement. J Clin Oncol 2004; 22: 3345-9.

19. Vuylsteke RJ, Borgstein PJ, van Leeuwen PA, Gietema HA, Molenkamp BG, Statius Muller MG, et al. Sentinel lymph node tumor load: an independent predictor of additional lymph node involvement and survival in melanoma. Ann Surg Oncol 2005; 12: 440-8. 